\title{
Basic science: (January 2008)
}

1. Abukhdeir AM, Vitolol MI, Argani P, De Marzo AM, Karakas B, Konishi H, Gustin JP, Lauring J, Garay JP, Pendleton C, Konishi Y, Blair BG, Brenner K, Garrett-Mayer E, Carraway H, Bachman KE, Park BH. Tamoxifen-stimulated growth of breast cancer due to p21 loss. Proc Natl Acad Sci USA 2008; 105: 288-293.

2. Allred DC, Wu Y, Mao S, Nagtegaal ID, Lee $S$, Perou CM, Mohsin SK, O'Connell P, Tsimelzon A, Medina D. Ductal carcinoma in situ and the emergence of diversity during breast cancer evolution. Clin Cancer Res 2008; 14: 370-378.

3. Arendt LM, Schuler LA. Prolactin drives estrogen receptor- $\alpha$-dependent ductal expansion and synergizes with transforming growth factor- $\alpha$ to induce mammary tumors in males. $A m$ J Pathol 2008; 172: 194-202.

4. Arlander SJH, Greene BT, Innes CL, Paules RS. DNA protein kinase-dependent G(2) checkpoint revealed following knockdown of ataxia-telangiectasia mutated in human mammary epithelial cells. Cancer Res 2008; 68: 89-97.

5. Begg CB, Haile RW, Borg A, Malone KE, Concannon P, Thomas DC, Langholz B, Bernstein L, Olsen JH, Lynch CF, Anton-Culver H, Capanu $M$, Liang XL, Hummer AJ, Sima C, Bernstein JL. Variation of breast cancer risk among BRCA1/2 carriers. JAMA 2008; 299: 194-201.

6. Bollet MA, Servant N, Neuvial P, Decraene C, Lebigot I, Meyniel JP, De Rycke Y, Savignoni A, Rigaill G, Hupe P, Fourquet A, Sigal-Zafrani B, Barillot $E$, Thiery JP. High-resolution mapping of DNA breakpoints to define true recurrences among ipsilateral breast cancers. J Natl Cancer Inst 2008; 100: 48-58.

7. Brantley-Sieders DM, Zhuang G, Hicks D, Bin Fang W, Hwang Y, Cates JMM, Coffman K, Jackson D, Bruckheirner E, Muraoka-Cook RS, Chen J. The receptor tyrosine kinase EphA2 promotes mammary adenocarcinoma tumorigenesis and metastatic progression in mice by amplifying ErbB2 signaling. J Clin Invest 2008; 118: 64-78.
8. Bretschneider N, Brand H, Miller N, Lowery AJ, Kerin MJ, Gannon F, Denger S. Estrogen induces repression of the breast cancer and salivary gland expression gene in an estrogen receptor $\alpha$-dependent manner. Cancer Res 2008; 68: 106-114.

9. Bultman SJ, Herschkowitz JI, Godfrey V, Gebuhr TC, Yaniv M, Perou CM, Magnuson T. Characterization of mammary tumors from Brg1 heterozygous mice. Oncogene 2008; 27: 460-468.

10. Caldon C, Lee C, Sutherland R, Musgrove E. Wilms' tumor protein 1: an early target of progestin regulation in T-47D breast cancer cells that modulates proliferation and differentiation. Oncogene 2008; 27: 126-138.

11. Cascio S, Bartella V, Auriemma A, Johannes GJ, Russo A, Giordano A, Surmacz E. Mechanism of leptin expression in breast cancer cells: role of hypoxia-inducible factor- $1 \alpha$. Oncogene 2008; 27: 540-547.

12. Chakraborty G, Jain S, Kundu GC. Osteopontin promotes vascular endothelial growth factordependent breast tumor growth and angiogenesis via autocrine and paracrine mechanisms. Cancer Res 2008; 68: 152-161.

13. Chang TW, Chen CC, Chen KY, Su JH, Chang $\mathrm{JH}$, Chang MC. Ribosomal phosphoprotein PO interacts with GCIP and overexpression of PO is associated with cellular proliferation in breast and liver carcinoma cells. Oncogene 2008; 27: 332-338.

14. Debies MT, Gest SA, Mathers JL, Mikse OR, Leonard TL, Moody SE, Chodosh LA, Cardiff RD, Gunther EJ. Tumor escape in a Wnt1-dependent mouse breast cancer model is enabled by p19(Arf)/p53 pathway lesions but not p16(Ink4a) loss. J Clin Invest 2008; 118: 51-63.

15. Eckstein N, Servan K, Girard L, Cai D, von Jonquieres G, Jaehde U, Kassack MU, Gazdar AF, Minna JD, Royer HD. Epidermal growth factor receptor pathway analysis identifies amphiregulin as a key factor for cisplatin resistance of human breast cancer cells. J Biol Chem 2008; 283: 739-750. 
16. Frankel LB, Christoffersen NR, Jacobsen A, Lindow M, Krogh A, Lund AH. Programmed cell death 4 (PDCD4) is an important functional target of the microRNA miR-21 in breast cancer cells. J Biol Chem 2008; 283: 1026-1033.

17. Frietze $S$, Lupien $M$, Silver PA, Brown $M$. CARM1 regulates estrogen-stimulated breast cancer growth through up-regulation of E2F1. Cancer Res 2008; 68: 301-306.

18. Gao BN, Xie XJ, Huang CX, Shames DS, Chen TTL, Lewis CM, Bian AH, Zhang BF, Olopade OI, Garber JE, Euhus DM, Tomlinson GE, Minna JD. RASSF1A polymorphism A133S is associated with early onset breast cancer in BRCA1/2 mutation carriers. Cancer Res 2008; 68: 22-25.

19. Husemann $Y$, Geigl JB, Schubert F, Musiani $P$, Meyer M, Burghart E, Forni G, Eils R, Fehm T, RiethmUller G, Klein CA. Systemic spread is an early step in breast cancer. Cancer Cell 2008; 13: $58-68$.

20. Maraqa L, Cummings $M$, Peter MB, Shaaban AM, Horgan K, Hanby AM, Speirs V. Carcinoembryonic antigen cell adhesion molecule 6 predicts breast cancer recurrence following adjuvant tamoxifen. Clin Cancer Res 2008; 14: 405-411.

21. Meijnen P, Peterse JL, Antonini N, Rutgers E, de Vijver MJ. Immunohistochemical categorisation of ductal carcinoma in situ of the breast. Br J Cancer 2008; 98: 137-142.

22. Morishige M, Hashimoto S, Ogawa E, Toda $Y$, Kotani $H$, Hirose $M$, Wei $S$, Hashimoto $A$, Yamada A, Yano H, Mazaki Y, Kodama H, Nio $\mathrm{Y}$, Manabe T, Wada H, Kobayashi H, Sabe H. GeP100 links epidermal growth factor receptor signalling to Arf6 activation to induce breast cancer invasion. Nat Cell Biol 2008; 10: 85-U70.

23. Newman SP, Foster PA, Stengel C, Day JM, Ho YT, Judde JG, Lassalle M, Prevost G, Leese MP, Potter BVL, Reed MJ, Purohit A. STX140 is efficacious in vitro and in vivo in taxaneresistant breast carcinoma cells. Clin Cancer Res 2008; 14: 597-606.

24. Richard S, Vogel G, Huot ME, Guo T, Muller WJ, Lukong KE. Sam68 haploinsufficiency delays onset of mammary tumorigenesis and metastasis. Oncogene 2008; 27: 548-556.

25. Saal LH, Gruvberger-Saal SK, Persson C, Loevgren K, Jumppanen M, Staaf J, Jonsson G, Pires MM, Maurer M, Holm K, Koujak S, Subramaniyam S, Vallon-Christersson J, Olsson $\mathrm{H}$, Su T, Memeo L, Ludwig T, Ethier SP, Krogh M, Szabolcs M, Murty VV, Isola J, Hibshoosh H, Parsons R, Borg A. Recurrent gross mutations of the PTEN tumor suppressor gene in breast cancers with deficient DSB repair. Nat Genet 2008; 40: 102-107.

26. Shen $Q$, Uray IP, Li Y, Krisko TI, Strecker TE, Kim HT, Brown PH. The AP-1 transcription factor regulates breast cancer cell growth via cyclins and E2F factors. Oncogene 2008; 27: 366-377.

27. Storci G, Sansone P, Trere D, Tavolari S, Taffurelli M, Ceccarelli C, Guarnieri T, Paterini P, Pariali M, Montanaro L, Santini D, Chieco P, Bonafe $M$. The basal-like breast carcinoma phenotype is regulated by SLUG gene expression. J Pathol 2008; 214: 25-37.

28. Tan BK, Tan LK, Yu K, Tan PH, Lee M, Sii LH, Wong CY, Ho GH, Yeo AWY, Chow PKH, Koong HN, Yong WS, Lim DTH, Ooi L, Soo KC, Tan P. Clinical validation of a customized multiple signature microarray for breast cancer. Clin Cancer Res 2008; 14: 461-469.

29. Tavazoie SF, Alarcon C, Oskarsson T, Padua D, Wang QQ, Bos PD, Gerald WL, Massague J. Endogenous human microRNAs that suppress breast cancer metastasis. Nature 2008; 451: 147-U3.

30. Yang L, Huang JH, Ren XB, Gorska AE, Chytil A, Aakre $M$, Carbone DP, Matrisian LM, Richmond A, Lin PC, Mosesl HL. Abrogation of TGF $\beta$ signaling in mammary carcinomas recruits Gr-1+CD11b+ myeloid cells that promote metastasis. Cancer Cell 2008; 13: 23-35.

Prepared by

$R$ Sutherland

Cancer Research Program Garvan Institute of Medical Research Darlinghurst, NSW, Australia 